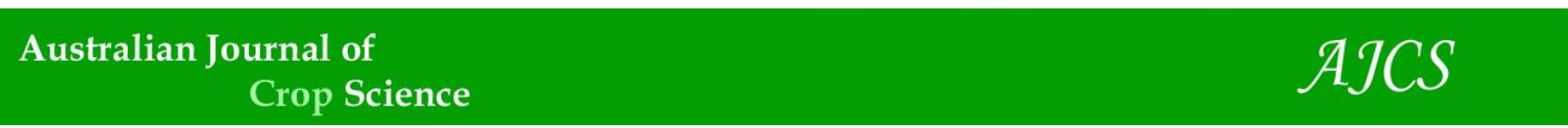

AJCS 14(11):1728-1735 (2020)

ISSN:1835-2707

doi: 10.21475/ajcs.20.14.11.p2323

\title{
Reference standards for soils cultivated with Urochloa brizantha and its use in nutritional diagnosis
}

\author{
André Cayô Cavalcanti ${ }^{1}$, Fábio Luiz Partelli ${ }^{1,3^{*}}$, Ivoney Gontijo ${ }^{1}$, Julien Chiquieri ${ }^{1}$, Heder Braun ${ }^{2}$ \\ ${ }^{1}$ Federal University of Espírito Santo, Center of North Espírito Santo (UFES-CEUNES), Departament Plant and Biology \\ Science, São Mateus, Espírito Santo State, Brazil \\ ${ }^{2}$ State University of Maranhão, Departament Plant Science and Pathology, Graduate Program in Agroecology, \\ Campus Universitário Paulo VI, s/n, Tirirical, São Luís, São Luís, Maranhão State, Brazil \\ ${ }^{3}$ Fellow of Productivity in Research, CNPq, Brazil
}

\section{*Corresponding author: partelli@yahoo.com.br}

\begin{abstract}
The Diagnosis and Recommendation Integrated System (DRIS) is a tool to assess the nutritional state of plants. Due to the decrease of soil fertility in pasture areas and little information about fertilization recommendations, the nutritional reference norms for soil and sufficiency range can be useful tools to help future fertilization. Norms DRIS has been proved efficient as a method for nutritional diagnosis in several crops. However, there are a lack of information on the use of DRIS and sufficiency range for Latosols and Acrisols cultivated with Urochloa brizantha. Thus, the objective of the present study was to establish reference nutritional norms using sufficiency ranges and DRIS norms for Latosols and Acrisols cultivated with Urochloa brizantha and their use in nutritional diagnosis. Soils samples from 20 Urochloa brizantha pastures sites of the North of Espírito Santo State were used to establish the reference norms, and a further 85 soils samples were randomly collected for diagnosis analysis, in order to characterize nutritional condition of pastures. DRIS norms and sufficiency ranges were established for Latosols and Acrisols cultivated with $\mathrm{U}$. brizantha pastures. The differences found between soil norms for other Brazilian regions allow us to infer that the norms should be used only in the conditions in which they were developed. Our results suggest that using DRIS norms and sufficiency ranges developed on the basis of soil analysis revealed deficiency of $\mathrm{P}, \mathrm{B}, \mathrm{Cu}$, and $\mathrm{Zn}$ in more than $40 \%$ of the pastures cultivated with $U$. brizantha and $47 \%$ of the areas needed liming.
\end{abstract}

Keywords: Pasture, Mineral Nutrition, Sufficiency range, DRIS.

Abbreviations: OM_organic matter, Ca_calcium, Mg_magnesium, K_potassium, P_phosphorus, S_sulfur, Zn_zinc, B_boron, Cu_copper, Fe_iron, Mn_manganese, V_base saturation, T_cation exchange capacity at pH 7.

\section{Introduction}

Agribusiness represents about $25 \%$ of the Brazilian Gross Domestic Product being a source of wealth for the country, and it generates thousands of jobs. Livestock production systems accounts for $30 \%$ of this sector (ABIEC, 2016), and 167 million hectares of pastures are allocated to this activity (Embrapa, 2018).

Pasture areas in Brazil cultivated with species of the genus Urochloa have increased significantly compared to other forage crops. The specie $U$. brizantha, commonly known as "braquiarão", is one of the most planted crops because it is well adapted to soils with low or medium fertility, and it constitute a substantial part of the Brazilian pastures (Sousa et al., 2010). The main problems facing livestock rearing are climatic seasonality (found in a large part of the Brazilian regions) and the absence of pasture nutritional management (Cavalcanti et al., 2017). Then the extensive area destined to this activity is not used to its full potential, often culminating in the appearance of degraded pastures.

Most part of the cultivated pastures areas in Brazil are completly deteriorated or in process of degradation. The partial degradation is an evolutionary progress of loss of vigor and forage plant yield with no possibility of natural recovery, that affects animal production, performance and culminates in the degradation of the soil and natural resources due to improper management (Boddey et al., 2004; Oliveira et al., 2004; Santos et al., 2015). One of the main causes degradation of soil is the absence of soil fertility maintenance, because the nutrients extracted by the plant are not replaced. This problem could be solved by liming soils to maintain the appropriate $\mathrm{pH}$ for optimum nutrient supply, fertilizer application to maintain recommended levels of nutrients in soils and covering the soil with vegetation as much as it is practicable to retain all nutrients in soil. These are therefore an essential practice for increase of plant yield, and should be based on correct nutritional diagnosis specific to each cultivated species.

Soil chemical analysis are important to determine sources, quantities and the most suitable time for the producer to apply corrective measures and fertilizers. For this, the element content in the soil should be balanced with the plant characteristics cultivated in the field, such as growth rate, nutritional content and crop yield (Partelli et al., 2014). 
The critical level or sufficiency ranges are most widely used to establish the diagnosis of soil and leaf nutritional analysis because a specific plant part above which near maximal yield is produced and below which yield loss is expected (Dow and Roberts, 1982). According to Beaufils and Sumner (1976), the concept of nutrient balance contained in the Diagnosis and Recommendation Integrated System (DRIS) for leaf analysis also can be used for soil, increasing the options for interpreting the fertility of this component. The DRIS method has been demonstrated in studies on corn (Rocha et al., 2007), orange (Camacho et al., 2012; Dias et al., 2013), cotton (Morais et al., 2009), common beans (Mesquita et al., 2018), sugarcane (Guimarães et al., 2015), apple (Sofi et al., 2017) and coffee (Partelli et al., 2006; Cavalcanti et al., 2017).

Regional patterns may contribute to the rational use of agricultural chemicals, improving the plant nutritional balance and consequently, increasing the yield, and further reducing crop production costs (Cavalcanti et al., 2017; Dias et al., 2010).

No studies were found in the literature comprising the diagnosis of nutrients in the soil that limit the dry matter yield of $U$. brizantha using DRIS norms, sufficiency ranges and nutritional diagnosis. Thus, the objective of the present study was to establish nutritional reference norms, such as sufficiency ranges and DRIS norms, for Latosols and Argisolos cultivated with Urochloa brizantha and their use in nutritional diagnosis.

\section{Results and Discussion}

\section{DRIS norms and sufficiency ranges}

The norms DRIS, expressed by the proportion between two nutrients concentrations, their chemical characteristics obtained in the soil from $20 \mathrm{U}$. brizantha pastures were used for the soil fertility diagnosis by DRIS in pastures cultivated on Latosols and Acrisols (Table 1).

Beaufils and Sumner (1976) concluded that the technique of balancing nutrients is applicable to soils and plants, that increases the scope of DRIS action. Satisfactory results have been obtained using DRIS in the soil, as reported by Santana et al. (2008), Morais et al. (2009), and Cavalcanti et al. (2017).

In plants, nutrients are analyzed for total concentration and represent the nutrient concentrations and proportions that perform determined metabolic functions. Chemical soil analysis for the purpose of recommending fertilizers is a process in which quick methods are used to estimate the availability of nutrients and to simulate the extraction capacity of

roots. Then, o method DRIS applied in soils is important in fertilization management, because it is easier to alter nutrient concentrations in the soil, by liming or fertilization, than to alter the leaf concentrations (Dias et al., 2010, Santana et al., 2008).

In addition, Batista and Batista (2010) studied interference in nutrient supply in different grasses to determine the suitable nutrient supplement to the plants. Making an efficient use of pasture in intensive production systems, there should be proper quantities of nutrients in the forage dry matter (Hopkins et al., 1994). When grasses are fertilized, there may be an increase (especially) of a supplied nutrient, but there may also be secondary effects from this application, resulting in increases or decreases in the contents of other nutrients. Thus, applying a nutrient may benefit or harm the content and action of others (Whitehead, 2000).

\section{Nutritional diagnosis and interpretation classes of the chemical atributes of the soil}

The sufficiency ranges established for soil (Table 2) can be used to diagnose the fertility in $U$. brizantha pastures cultivated on Latosols and Acrisols. The mean of organic matter concentration in soil in productive pastures established as reference (Table 2) was classified as medium (Table 3) according to the Minas Gerais Soil Fertility Commission (Ribeiro et al., 1999). Costa Júnior et al. (2012) showed that the organic matter stability is more important in soil than its quantity itself. As organic fertilization is consolidated, the immobilization/mineralization balance tends to mineralization, thus supporting higher yields and supplying more stable nutrients to the plants.

It should be emphasized that the effect of soil organic matter on pasture yield can be direct through nutrient supply, or indirect through modifying the physical properties of the soil. Better soil physical conditions allow increasing retention capacity and circulation of the water in the soil, improving the root development and consequently stimulating forage plant growth and also decreasing the soil erosion (Costa Júnior et al., 2012). In tropical environments, most soil organic matter is formed by humic substances that contribute about 80 to $90 \%$ of the organic carbon in the soil, where the relative distribution of the humified fractions can be used as organic matter quality indicators (Partelli et al., 2009).

The phosphorus (P) available in the soil for plants is determined by several extraction methods. According to Steiner et al. (2012) some methods extracting $P$ from different soil types has greater or lesser ease. These methods can, in some clay soils, extract less $\mathrm{P}$ than the true soil content. This fact possibly was due to the $P$ concentration in soil obtained by resin techniques to be more reliable than the Mehlich method. The $P$ is classified as very low (Table 2), according to Raij et al. (1997) and the Minas Gerais Soil Fertility Commission (Ribeiro et al., 1999) (Table 3). It is known that $P$ has important functions in the initial development phase of forage plants, and it plays an important role in root system growth and grass tillering, that are essential for bigger forage plant productivity. This nutrient, is considered the second most limiting nutrient to plant growth and it is composed of nucleic acids, nucleotides, coenzymes, and sugar phosphates (Veneklaas et al., 2012). The $P$ is not always available to the plant, since the contents in the soil are relatively low. Part of this nutrient has covalent linkages with soil colloids and it is therefore fixed in most soils, especially in soils rich in iron sesquioxides and/or aluminum and acids (Oladiran et al., 2012), as it is the case of much of Brazilian soils.

Silva et al. (2013) showed that no difference were observed among the different $\mathrm{P}$ sources on dry matter production of Bracharia brizantha. These authors, considered all phosphorus sources and mencioned that phosphorus average rates to provide $90 \%$ of the maximum shoot dry matter production of forage grass was $408 \mathrm{mg} \mathrm{dm}^{-3}$. The use of phosphate fertilizer usually gives favorable results and improve forage plant tillering as the root system (Deminicies et al., 2010). 
Table 1. DRIS norms of the soil in pastures cultivated with U. brizantha on Latosols and Acrisols.

\begin{tabular}{|c|c|c|c|c|c|c|c|}
\hline Ratio & Mean & Standard deviation & $\mathrm{CV}(\%)$ & Ratio & Mean & Standard deviation & $\mathrm{CV}(\%)$ \\
\hline OM/P & 2.356 & 1.172 & 49.73 & $\mathrm{Cu} / \mathrm{MO}$ & 0.099 & 0.067 & 67.28 \\
\hline $\mathrm{OM} / \mathrm{K}$ & 0.202 & 0.069 & 34.05 & $\mathrm{Cu} / \mathrm{P}$ & 0.181 & 0.049 & 26.85 \\
\hline $\mathrm{OM} / \mathrm{Ca}$ & 10.68 & 3.557 & 33.29 & $\mathrm{Cu} / \mathrm{K}$ & 0.017 & 0.006 & 34.41 \\
\hline $\mathrm{OM} / \mathrm{Mg}$ & 38.21 & 15.26 & 39.95 & $\mathrm{Cu} / \mathrm{Ca}$ & 0.899 & 0.315 & 35.08 \\
\hline $\mathrm{OM} / \mathrm{S}$ & 1.840 & 0.752 & 40.86 & $\mathrm{Cu} / \mathrm{Mg}$ & 3.375 & 1.643 & 48.68 \\
\hline $\mathrm{OM} / \mathrm{B}$ & 177.12 & 67.91 & 38.34 & $\mathrm{Cu} / \mathrm{S}$ & 0.165 & 0.086 & 52.46 \\
\hline $\mathrm{OM} / \mathrm{Cu}$ & 13.47 & 6.335 & 47.01 & $\mathrm{Cu} / \mathrm{B}$ & 15.05 & 6.040 & 40.12 \\
\hline $\mathrm{OM} / \mathrm{Fe}$ & 0.872 & 0.288 & 33.07 & $\mathrm{Cu} / \mathrm{Fe}$ & 0.075 & 0.029 & 38.15 \\
\hline $\mathrm{OM} / \mathrm{Mn}$ & 1.586 & 0.726 & 45.76 & $\mathrm{Cu} / \mathrm{Mn}$ & 0.140 & 0.066 & 47.37 \\
\hline $\mathrm{OM} / \mathrm{Zn}$ & 7.582 & 5.911 & 77.96 & $\mathrm{Cu} / \mathrm{Zn}$ & 0.537 & 0.164 & 30.56 \\
\hline OM/BS & 0.434 & 0.118 & 27.21 & $\mathrm{Cu} / \mathrm{BS}$ & 0.037 & 0.013 & 35.97 \\
\hline OM/CEC & 4.274 & 1.263 & 29.56 & $\mathrm{Cu} / \mathrm{CEC}$ & 0.368 & 0.141 & 38.45 \\
\hline $\mathrm{P} / \mathrm{OM}$ & 0.560 & 0.374 & 66.73 & $\mathrm{Fe} / \mathrm{OM}$ & 1.301 & 0.537 & 41.28 \\
\hline $\mathrm{P} / \mathrm{K}$ & 0.097 & 0.029 & 29.85 & $\mathrm{Fe} / \mathrm{P}$ & 2.888 & 1.932 & 66.91 \\
\hline $\mathrm{P} / \mathrm{Ca}$ & 5.142 & 1.661 & 32.31 & $\mathrm{Fe} / \mathrm{K}$ & 0.233 & 0.036 & 15.59 \\
\hline $\mathrm{P} / \mathrm{Mg}$ & 19.10 & 8.294 & 43.42 & $\mathrm{Fe} / \mathrm{Ca}$ & 12.91 & 4.953 & 38.37 \\
\hline $\mathrm{P} / \mathrm{S}$ & 0.912 & 0.423 & 46.43 & $\mathrm{Fe} / \mathrm{Mg}$ & 44.77 & 13.13 & 29.32 \\
\hline $\mathrm{P} / \mathrm{B}$ & 86.17 & 32.73 & 37.99 & $\mathrm{Fe} / \mathrm{S}$ & 2.305 & 1.211 & 52.55 \\
\hline $\mathrm{P} / \mathrm{Cu}$ & 5.881 & 1.424 & 24.21 & $\mathrm{Fe} / \mathrm{B}$ & 206.7 & 55.79 & 27.00 \\
\hline $\mathrm{P} / \mathrm{Fe}$ & 0.430 & 0.146 & 34.03 & $\mathrm{Fe} / \mathrm{Cu}$ & 15.91 & 7.772 & 48.86 \\
\hline $\mathrm{P} / \mathrm{Mn}$ & 0.803 & 0.364 & 45.36 & $\mathrm{Fe} / \mathrm{Mn}$ & 1.821 & 0.498 & 27.33 \\
\hline $\mathrm{P} / \mathrm{Zn}$ & 3.145 & 1.533 & 48.72 & $\mathrm{Fe} / \mathrm{Zn}$ & 9.151 & 7.670 & 83.81 \\
\hline $\mathrm{P} / \mathrm{BS}$ & 0.211 & 0.066 & 31.06 & $\mathrm{Fe} / \mathrm{BS}$ & 0.523 & 0.152 & 29.00 \\
\hline $\mathrm{P} / \mathrm{CEC}$ & 2.090 & 0.704 & 33.70 & $\mathrm{Fe} / \mathrm{CEC}$ & 5.074 & 1.290 & 25.42 \\
\hline $\mathrm{K} / \mathrm{OM}$ & 5.724 & 2.654 & 46.37 & $\mathrm{Mn} / \mathrm{OM}$ & 0.782 & 0.372 & 47.59 \\
\hline $\mathrm{K} / \mathrm{P}$ & 11.96 & 6.172 & 51.63 & $\mathrm{Mn} / \mathrm{P}$ & 1.841 & 1.465 & 79.60 \\
\hline $\mathrm{K} / \mathrm{Ca}$ & 54.61 & 13.87 & 25.39 & $\mathrm{Mn} / \mathrm{K}$ & 0.143 & 0.068 & 47.15 \\
\hline $\mathrm{K} / \mathrm{Mg}$ & 192.3 & 48.87 & 25.41 & $\mathrm{Mn} / \mathrm{Ca}$ & 7.760 & 3.808 & 49.07 \\
\hline $\mathrm{K} / \mathrm{S}$ & 9.866 & 4.434 & 44.94 & $\mathrm{Mn} / \mathrm{Mg}$ & 24.79 & 4.110 & 16.58 \\
\hline $\mathrm{K} / \mathrm{B}$ & 896.2 & 240.8 & 26.87 & $\mathrm{Mn} / \mathrm{S}$ & 1.423 & 0.892 & 62.70 \\
\hline $\mathrm{K} / \mathrm{Cu}$ & 66.52 & 23.87 & 35.88 & $\mathrm{Mn} / \mathrm{B}$ & 120.4 & 40.015 & 33.25 \\
\hline $\mathrm{K} / \mathrm{Fe}$ & 4.392 & 0.708 & 16.11 & $\mathrm{Mn} / \mathrm{Cu}$ & 10.26 & 7.552 & 73.58 \\
\hline $\mathrm{K} / \mathrm{Mn}$ & 7.945 & 2.328 & 29.30 & $\mathrm{Mn} / \mathrm{Fe}$ & 0.619 & 0.286 & 46.25 \\
\hline $\mathrm{K} / \mathrm{Zn}$ & 37.11 & 24.34 & 65.57 & $\mathrm{Mn} / \mathrm{Zn}$ & 6.139 & 6.121 & 99.71 \\
\hline K/BS & 2.229 & 0.402 & 18.06 & $\mathrm{Mn} / \mathrm{BS}$ & 0.312 & 0.124 & 39.69 \\
\hline $\mathrm{K} / \mathrm{CEC}$ & 21.74 & 3.724 & 17.13 & $\mathrm{Mn} / \mathrm{CEC}$ & 2.979 & 0.959 & 32.20 \\
\hline $\mathrm{Ca} / \mathrm{OM}$ & 0.106 & 0.043 & 40.56 & $\mathrm{Zn} / \mathrm{OM}$ & 0.198 & 0.131 & 65.95 \\
\hline $\mathrm{Ca} / \mathrm{P}$ & 0.222 & 0.101 & 45.62 & $\mathrm{Zn} / \mathrm{P}$ & 0.356 & 0.097 & 27.11 \\
\hline $\mathrm{Ca} / \mathrm{K}$ & 0.019 & 0.004 & 20.96 & $\mathrm{Zn} / \mathrm{K}$ & 0.034 & 0.013 & 37.03 \\
\hline $\mathrm{Ca} / \mathrm{Mg}$ & 3.642 & 1.038 & 28.49 & $\mathrm{Zn} / \mathrm{Ca}$ & 1.788 & 0.644 & 36.00 \\
\hline $\mathrm{Ca} / \mathrm{S}$ & 0.186 & 0.087 & 46.77 & $\mathrm{Zn} / \mathrm{Mg}$ & 6.918 & 3.373 & 48.76 \\
\hline $\mathrm{Ca} / \mathrm{B}$ & 17.14 & 5.354 & 31.24 & $\mathrm{Zn} / \mathrm{S}$ & 0.322 & 0.170 & 52.86 \\
\hline $\mathrm{Ca} / \mathrm{Cu}$ & 1.247 & 0.440 & 35.31 & $\mathrm{Zn} / \mathrm{B}$ & 31.36 & 15.53 & 49.53 \\
\hline $\mathrm{Ca} / \mathrm{Fe}$ & 0.085 & 0.022 & 25.37 & $\mathrm{Zn} / \mathrm{Cu}$ & 2.025 & 0.603 & 29.76 \\
\hline $\mathrm{Ca} / \mathrm{Mn}$ & 0.152 & 0.053 & 34.73 & $\mathrm{Zn} / \mathrm{Fe}$ & 0.153 & 0.065 & 42.10 \\
\hline $\mathrm{Ca} / \mathrm{Zn}$ & 0.674 & 0.364 & 53.92 & $\mathrm{Zn} / \mathrm{Mn}$ & 0.292 & 0.150 & 51.26 \\
\hline $\mathrm{Ca} / \mathrm{BS}$ & 0.042 & 0.006 & 13.79 & $\mathrm{Zn} / \mathrm{BS}$ & 0.074 & 0.027 & 36.59 \\
\hline $\mathrm{Ca} / \mathrm{CEC}$ & 0.408 & 0.064 & 15.79 & $\mathrm{Zn} / \mathrm{CEC}$ & 0.743 & 0.305 & 40.98 \\
\hline $\mathrm{Mg} / \mathrm{OM}$ & 0.032 & 0.016 & 48.96 & $\mathrm{BS} / \mathrm{OM}$ & 2.525 & 0.905 & 35.84 \\
\hline $\mathrm{Mg} / \mathrm{P}$ & 0.071 & 0.048 & 67.74 & $\mathrm{BS} / \mathrm{P}$ & 5.339 & 2.188 & 40.97 \\
\hline $\mathrm{Mg} / \mathrm{K}$ & 0.006 & 0.002 & 42.01 & $\mathrm{BS} / \mathrm{K}$ & 0.462 & 0.079 & 17.20 \\
\hline $\mathrm{Mg} / \mathrm{Ca}$ & 0.309 & 0.134 & 43.38 & $\mathrm{BS} / \mathrm{Ca}$ & 24.46 & 3.542 & 14.48 \\
\hline $\mathrm{Mg} / \mathrm{S}$ & 0.056 & 0.029 & 52.20 & $\mathrm{BS} / \mathrm{Mg}$ & 87.36 & 23.80 & 27.24 \\
\hline $\mathrm{Mg} / \mathrm{B}$ & 4.872 & 1.326 & 27.23 & $\mathrm{BS} / \mathrm{S}$ & 4.372 & 1.612 & 36.87 \\
\hline $\mathrm{Mg} / \mathrm{Cu}$ & 0.406 & 0.278 & 68.38 & $\mathrm{BS} / \mathrm{B}$ & 407.6 & 108.2 & 26.55 \\
\hline $\mathrm{Mg} / \mathrm{Fe}$ & 0.025 & 0.011 & 43.83 & $\mathrm{BS} / \mathrm{Cu}$ & 30.32 & 10.72 & 35.36 \\
\hline $\mathrm{Mg} / \mathrm{Mn}$ & 0.041 & 0.007 & 16.01 & $\mathrm{BS} / \mathrm{Fe}$ & 2.030 & 0.439 & 21.63 \\
\hline $\mathrm{Mg} / \mathrm{Zn}$ & 0.235 & 0.211 & 89.85 & $\mathrm{BS} / \mathrm{Mn}$ & 3.638 & 1.205 & 33.14 \\
\hline $\mathrm{Mg} / \mathrm{V}$ & 0.012 & 0.004 & 33.96 & $\mathrm{BS} / \mathrm{Zn}$ & 16.52 & 9.385 & 56.83 \\
\hline $\mathrm{Mg} / \mathrm{CEC}$ & 0.119 & 0.030 & 24.85 & $\mathrm{BS} / \mathrm{CEC}$ & 9.825 & 1.204 & 12.25 \\
\hline S/OM & 0.624 & 0.228 & 36.48 & CEC/OM & 0.262 & 0.106 & 40.57 \\
\hline $\mathrm{S} / \mathrm{P}$ & 1.351 & 0.665 & 49.19 & $\mathrm{CEC} / \mathrm{P}$ & 0.558 & 0.263 & 47.20 \\
\hline $\mathrm{S} / \mathrm{K}$ & 0.121 & 0.051 & 42.63 & $\mathrm{CEC} / \mathrm{K}$ & 0.047 & 0.009 & 19.25 \\
\hline $\mathrm{S} / \mathrm{Ca}$ & 6.418 & 2.693 & 41.95 & $\mathrm{CEC} / \mathrm{Ca}$ & 2.525 & 0.522 & 20.68 \\
\hline $\mathrm{S} / \mathrm{Mg}$ & 23.37 & 13.00 & 55.63 & $\mathrm{CEC} / \mathrm{Mg}$ & 8.806 & 1.881 & 21.36 \\
\hline S/B & 106.9 & 57.63 & 53.90 & $\mathrm{CEC} / \mathrm{S}$ & 0.451 & 0.168 & 37.37 \\
\hline $\mathrm{S} / \mathrm{Cu}$ & 7.968 & 4.447 & 55.82 & $\mathrm{CEC} / \mathrm{B}$ & 41.416 & 9.222 & 22.27 \\
\hline $\mathrm{S} / \mathrm{Fe}$ & 0.525 & 0.222 & 42.32 & $\mathrm{CEC} / \mathrm{Cu}$ & 3.158 & 1.304 & 41.28 \\
\hline $\mathrm{S} / \mathrm{Mn}$ & 0.987 & 0.623 & 63.11 & $\mathrm{CEC} / \mathrm{Fe}$ & 0.208 & 0.046 & 22.26 \\
\hline$S / Z n$ & 4.143 & 2.446 & 59.05 & $\mathrm{CEC} / \mathrm{Mn}$ & 0.366 & 0.103 & 28.13 \\
\hline S/BS & 0.258 & 0.091 & 35.29 & $\mathrm{CEC} / \mathrm{Zn}$ & 1.755 & 1.123 & 63.99 \\
\hline S/CEC & 2.541 & 1.016 & 39.97 & $\mathrm{CEC} / \mathrm{BS}$ & 0.103 & 0.013 & 12.37 \\
\hline $\mathrm{B} / \mathrm{OM}$ & 0.0067 & 0.0031 & 47.21 & $\mathrm{~B} / \mathrm{Cu}$ & 0.0799 & 0.0390 & 48.84 \\
\hline $\mathrm{B} / \mathrm{P}$ & 0.0142 & 0.0077 & 54.33 & $\mathrm{~B} / \mathrm{Fe}$ & 0.0052 & 0.0015 & 28.53 \\
\hline $\mathrm{B} / \mathrm{K}$ & 0.0012 & 0.0003 & 27.08 & $\mathrm{~B} / \mathrm{Mn}$ & 0.0090 & 0.0025 & 27.32 \\
\hline $\mathrm{B} / \mathrm{Ca}$ & 0.0650 & 0.0232 & 35.74 & $\mathrm{~B} / \mathrm{Zn}$ & 0.0458 & 0.0338 & 73.86 \\
\hline $\mathrm{B} / \mathrm{Mg}$ & 0.2205 & 0.0614 & 27.83 & $\mathrm{~B} / \mathrm{BS}$ & 0.0026 & 0.0007 & 26.07 \\
\hline $\mathrm{B} / \mathrm{S}$ & 0.0114 & 0.0046 & 40.12 & $\mathrm{~B} / \mathrm{CEC}$ & 0.0254 & 0.0058 & 22.88 \\
\hline
\end{tabular}

$\mathrm{OM}$ : Organic matter, V: base saturation, CEC: Cationic Exchange Capacity at pH 7.0, BS: base saturation, CV\% denote coefficient of variation. 

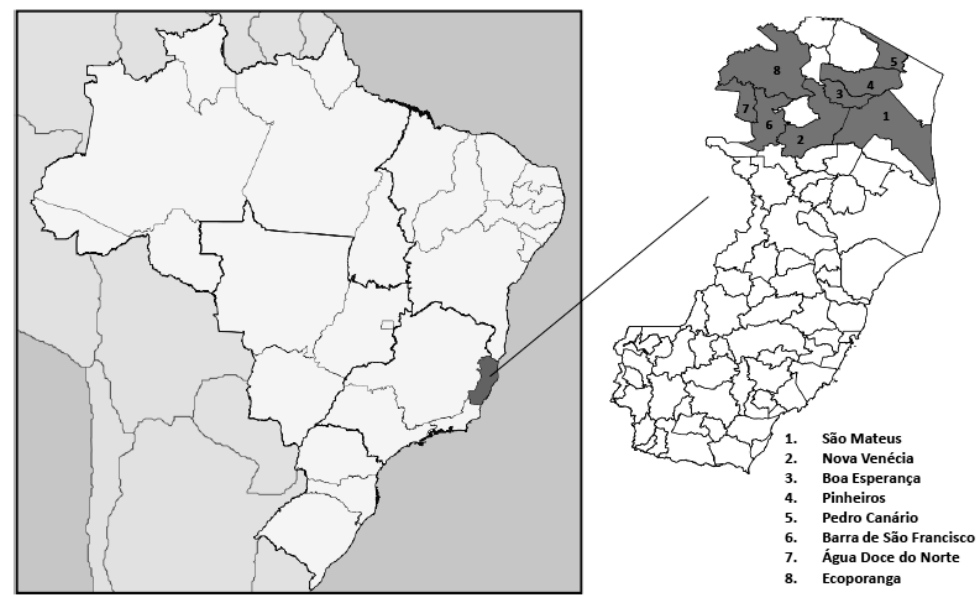

Fig 1. Schematic map showing areas where soil samples were collected from Urochloa brizantha pastures.

Table 2. Mean, standard deviation, sufficiency ranges and coefficient of variation (CV) of nutrients available in soil in productive pastures established as reference for Urochloa brizantha.

\begin{tabular}{|c|c|c|c|c|}
\hline Nutrients & Mean & Standard deviation & Sufficiency ranges & CV (\%) \\
\hline OM (dag dm ${ }^{-3}$ ) & 2.27 & 0.37 & $1.6-3.1$ & 16.54 \\
\hline$P\left(\mathrm{mg} \mathrm{dm}^{-3}\right)^{1}$ & 9.84 & 5.71 & $4.0-26.0$ & 58.00 \\
\hline$P\left(m g d m^{-3}\right)^{2}$ & 43.30 & 5.03 & $35.0-53.0$ & 11.61 \\
\hline $\mathrm{K}\left(\mathrm{mg} \mathrm{dm}^{-3}\right)$ & 67.40 & 27.87 & $25.0-120.0$ & 41.35 \\
\hline $\mathrm{Ca}\left(\mathrm{cmol}_{\mathrm{c}} \mathrm{dm}^{3}\right)$ & 1.85 & 0.59 & $1.0-3.0$ & 31.82 \\
\hline $\mathrm{Mg}\left(\mathrm{cmol}_{\mathrm{c}} \mathrm{dm}^{3}\right)$ & 0.58 & 0.17 & $0.3-0.8$ & 28.85 \\
\hline $\mathrm{S}\left(\mathrm{mg} \mathrm{dm}^{-3}\right)$ & 18.55 & 5.60 & $11.0-30.0$ & 30.17 \\
\hline$B\left(\mathrm{mg} \mathrm{dm}^{-3}\right)$ & 1.06 & 0.18 & $0.93-1.53$ & 16.77 \\
\hline $\mathrm{Cu}\left(\mathrm{mg} \mathrm{dm}^{-3}\right)$ & 0.44 & 0.31 & $0.2-1.3$ & 70.93 \\
\hline $\mathrm{Fe}\left(\mathrm{mg} \mathrm{dm^{-3 } )}\right.$ & 94.15 & 37.36 & $43-181$ & 39.69 \\
\hline $\mathrm{Mn}\left(\mathrm{mg} \mathrm{dm}^{-3}\right)$ & 28.00 & 11.97 & $11.0-48.0$ & 42.76 \\
\hline $\mathrm{Zn}\left(\mathrm{mg} \mathrm{dm} \mathrm{m}^{-3}\right)$ & 2.31 & 1.03 & $0.7-4.7$ & 49.16 \\
\hline BS (\%) & 66.60 & 7.62 & $58.06-72.96$ & 13.00 \\
\hline $\operatorname{CEC}\left(\mathrm{cmol}_{\mathrm{c}} \mathrm{dm}^{3}\right)$ & 4.78 & 0.88 & $3.80-6.43$ & 18.31 \\
\hline
\end{tabular}

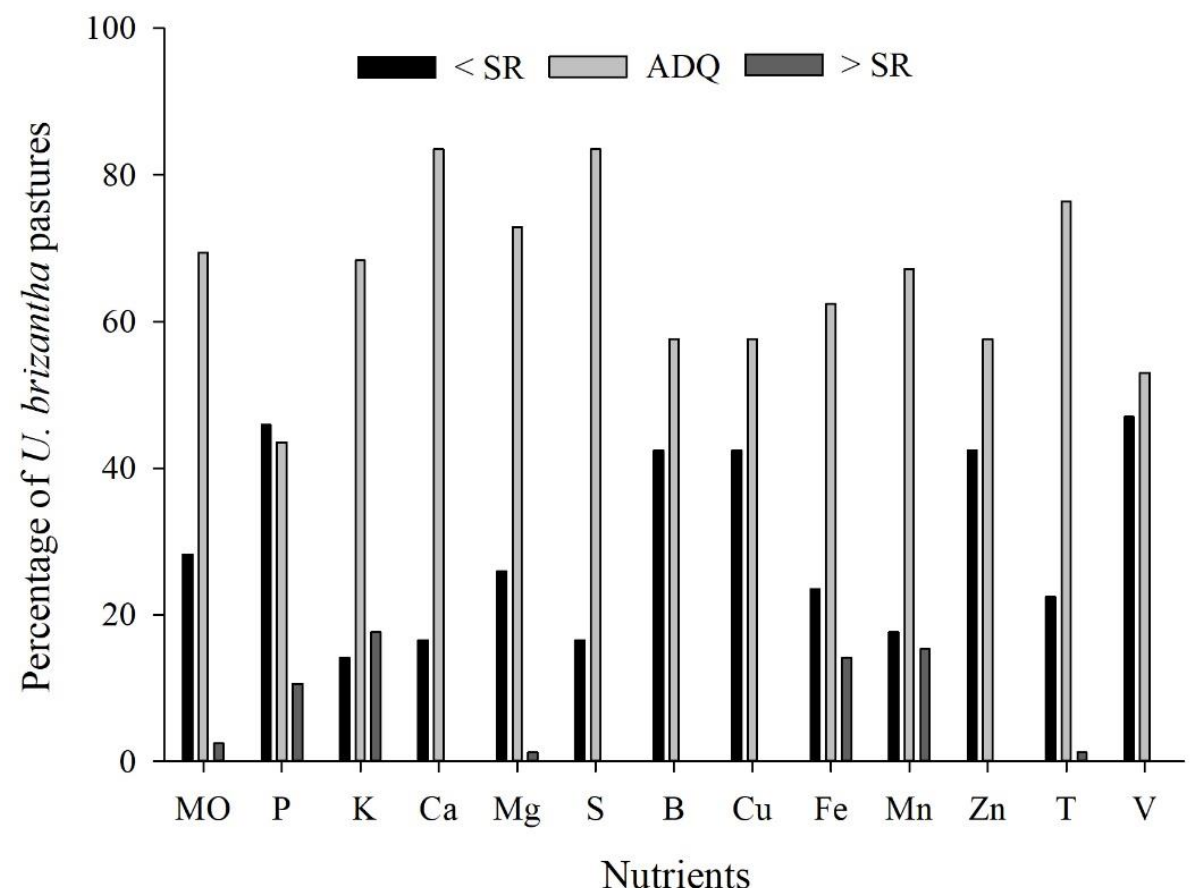

Fig 2. Percentage of $U$. brizantha pastures classified by the sufficiency range (SR) method for each nutrient as deficient (<SR), adequate (ADQ) and excessive (>SR). 
Table 3. Interpretation classes of the chemical atributes of the soil for $U$. brizantha pastures according to the Minas Gerais Soil Fertility Commission.

\begin{tabular}{|c|c|c|c|c|c|}
\hline \multirow{2}{*}{ Variável } & \multicolumn{5}{|c|}{ Interpretation classes } \\
\hline & Very low & Low & Medium & Adequada & High \\
\hline $\mathrm{OM}\left(\text { dag } \mathrm{dm}^{-3}\right)^{1}$ & $<0.70$ & $0.71-2.0$ & $2.01-4.0$ & $4.01-7.0$ & $>7.0$ \\
\hline$P\left(m g ~ d m^{-3}\right)^{1}$ & $<11.0$ & $11.1-15.8$ & $15.9-21.8$ & 21.9-33.0 & $>33.0$ \\
\hline$P\left(m g d^{-3}\right)^{2}$ & $<5.0$ & $6.0-12.0$ & $13.0-20.0$ & $21.0-30.0$ & $>30.0$ \\
\hline $\mathrm{K}(\mathrm{mg} \mathrm{dm})^{-3}$ & $<15.0$ & $16.0-40.0$ & $41.0-70.0$ & $71.0-120.0$ & $>120.0$ \\
\hline $\mathrm{Ca}\left(\mathrm{cmol}_{\mathrm{c}} \mathrm{dm}^{-3}\right)^{1}$ & $<0.40$ & $0.41-1.2$ & $1.21-2.4$ & $2.41-4.0$ & $>4.0$ \\
\hline $\mathrm{Mg}\left(\mathrm{cmol}_{\mathrm{c}} \mathrm{dm}^{-3}\right)^{1}$ & $<0.15$ & $0.16-0.45$ & $0.46-0.9$ & $0.91-1.5$ & $>1.5$ \\
\hline $\left.\mathrm{S}(\mathrm{mg} \mathrm{dm})^{-3}\right)^{1}$ & $<6.4$ & $6.5-9.4$ & $9.5-13.0$ & 13.1-19.6 & $>19.6$ \\
\hline$B\left(m g d m^{-3}\right)^{1}$ & $<0.15$ & $0.16-0.35$ & $0.36-0.6$ & $0.61-0.9$ & $>0.9$ \\
\hline $\mathrm{B}\left(\mathrm{mg} \mathrm{dm} \mathrm{m}^{-3}\right)^{2}$ & - & $<0.20$ & $0.21-0.60$ & - & $>0.60$ \\
\hline $\mathrm{Cu}\left(\mathrm{mg} \mathrm{dm}^{-3}\right)^{1}$ & $<0.3$ & $0.4-0.7$ & $0.8-1.2$ & $1.3-1.8$ & $>1.8$ \\
\hline $\left.\mathrm{Cu}(\mathrm{mg} \mathrm{dm})^{-3}\right)^{2}$ & - & $<0.50$ & $0.6-0.8$ & - & $>0.8$ \\
\hline $\mathrm{Fe}\left(\mathrm{mg} \mathrm{dm} \mathrm{m}^{-3}\right)^{1}$ & $<8.0$ & $9.0-18.0$ & $19.0-30.0$ & $31.0-45.0$ & $>45.0$ \\
\hline $\mathrm{Fe}\left(\mathrm{mg} \mathrm{dm}^{-3}\right)^{2}$ & - & $<4.0$ & $5.0-12.0$ & - & $>12.0$ \\
\hline $\mathrm{Mn}\left(\mathrm{mg} \mathrm{dm}^{-3}\right)^{1}$ & $<2.0$ & $3.0-5.0$ & $6.0-8.0$ & $9.0-12.0$ & $>12.0$ \\
\hline $\mathrm{Mn}\left(\mathrm{mg} \mathrm{dm}^{-3}\right)^{2}$ & - & $<1.2$ & $1.3-5.0$ & - & $>5.0$ \\
\hline $\mathrm{Zn}\left(\mathrm{mg} \mathrm{dm}^{-3}\right)^{1}$ & $<0.4$ & $0.5-0.9$ & $1.0-1.5$ & $1.6-2.2$ & $>2.2$ \\
\hline $\mathrm{Zn}\left(\mathrm{mg} \mathrm{dm} \mathrm{m}^{-3}\right)^{2}$ & - & $<0.5$ & $0.6-1.2$ & - & $>1.2$ \\
\hline $\mathrm{BS}(\%)^{1}$ & $<20.0$ & $20.1-40.0$ & $40.1-60.0$ & $60.1-80.0$ & $>80.0$ \\
\hline $\operatorname{CEC}\left(\mathrm{cmol}_{\mathrm{C}} \mathrm{dm}^{-3}\right)^{1}$ & $<1.60$ & $1.61-4.3$ & $4.31-8.6$ & $8.61-15.0$ & $>15.0$ \\
\hline
\end{tabular}

\footnotetext{
${ }^{1}$ Interpreted by method of Ribeiro et al. (1999) and ${ }^{2}$ Interpreted by method of Raij et al. (1997).
}

According to the Minas Gerais Soil Fertility Commission (Ribeiro et al., 1999), the potassium (K) concentrations (Table 2) were classified as medium (Table 1). Forage grasses are relatively demanding in potassium, mainly in intensive pasture exploration systems (Martins et al., 2013). The potassium is a nutrient of agronomic interest, particularly for grassland, but it is not yet of serious environmental concern when compared with others nutrients as such nitrogen. The potassium is a structural element of soil minerals, and appears in three forms: as exchangeable ion adsorbed or released form to clay minerals and organic matter, and is present in the soil solution (Marschner, 1995). The loss of potassium by leaching from forage grass is usually low, but high levels of available soil $\mathrm{K}$, high $\mathrm{K}$ input from fertilizer or sandy soil with little clay content lead to increasing losses (Kayser and Isselstein, 2005). Then, nutrient cycling and leaching might influence the concentrations found of this nutrient in soil analysis (Garcia et al., 2008).

The calcium ( $\mathrm{Ca}$ ) and magnesium ( $\mathrm{Mg}$ ) concentrations (Table 2) observed in the areas under study are classified as medium (Table 3) according to the Minas Gerais Soil Fertility Commission (Ribeiro et al., 1999). The most viable way of supplying $\mathrm{Ca}$ and $\mathrm{Mg}$ to forage plants is by liming, applying limestone. This practice aims to supply $\mathrm{Ca}$ and $\mathrm{Mg}$ and neutralize soil acidity, by raising the soil base saturation, that varies according to the forage species (Pimenta et al., 2010). Limestone can favor the activity of primary macronutrients $(\mathrm{N}, \mathrm{P}$, and $\mathrm{K})$, maximizing their action in the soil. With this, the plant root system develops its capacity to absorb these nutrients existing in the fertilizer and water (Oliveira et al., 2009).

Practices such as liming, in addition to being essential for good pasture performance, can alter the nutrient dynamic and establish a balance in the soil-plant- animal system or further, improve these relationships for sustainability (Pimenta et al., 2010).

The sulfur (S) concentration (Table 2) was considered high (Table 3) according to the Minas Gerais Soil Fertility Commission (Ribeiro et al., 1999). Sulfur is found in an organic form in the soil, that presents more than $90 \%$ of the total nutrient content in most soils, and in the inorganic form. Sulfur is absorbed by plants in the form of $\mathrm{SO}_{4}{ }^{2-}$ and its main characteristic is the mobility in the soil, and it tends specially to concentrate in the surface layers and this fact is favored by the surface phosphorus concentration. Clay soils with high iron oxide contents have a large $\mathrm{SO}_{4}{ }^{2-}$ absorption capacity that decreases their movement in the soil profile. In sandy soils, the $\mathrm{SO}_{4}{ }^{2-}$ movement is bigger and thus it can be lost by percolation. Furthermore, sandy soils have low organic matter contents, and consequently smaller organic sulfur reserves (Tiwari, 2006).

There are reports that $S$ deficiency reaches about $50 \%$ of the total area of soils in Tropical America (Batista and Batista, 2010). When dealing with pastures with grass forage crops, sulfur is very important, because these types of plants demand this nutrient, especially when the $\mathrm{N}$ supply is high (Batista and Batista, 2010).

Regarding the micronutrients (Table 2 ), only copper ( $\mathrm{Cu}$ ) was classified as low; zinc ( $\mathrm{Zn})$ was considered suitable and $\mathrm{B}, \mathrm{Fe}$, and $\mathrm{Mn}$ were considered high (Table 1 ) according to Raij et al. (1997) and the Minas Gerais Soil Fertility Commission (Ribeiro et al., 1999).

Micronutrient deficiency is a limiting factor for yield and varies according to the type of crop and soil, and can result in a small reduction to complete loss of the production. Recently, concern about micronutrient deficiency has increased, due to their important role in resistance to stress and plant diseases, but the response to micronutrients by grass forage are not common.

Base saturation and cationic exchange capacity at $\mathrm{pH} 7.0$ (Table 2) were classified as medium (Table 3 ) by the Minas Gerais Soil Fertility Commission (Ribeiro et al., 1999). According to the Recommendation Manual for Liming and Fertilization for the State of Espírito Santo (Prezotti et al., 2007), soils cultivated with high yielding forage plants should have a basic saturation (BS, \%) over $60 \%$, and our results match with these findings. 
Regarding the nutritional diagnosis of the soil, it was observed that more than $40 \%$ of the pastures assessed presented $\mathrm{P}, \mathrm{B}, \mathrm{Cu}$ and $\mathrm{Zn}$ concentrations below the recommended (Figure 2). Verification of the base saturation also showed that there is need for liming in $47 \%$ of the areas. These problems could be solved by correcting though the fertilization with the limiting nutrients at levels within the sufficiency range recommended for the soil.

It is known that soil fertility and plant mineral nutrition management in pasture ecosystems is an essential tool to maintain the quality, productivity and perenniality of these areas. Reports are common in the literature of pasture degradation processes where the main causes include decreased soil fertility, especially with macro and micro nutrients, that negatively influence pasture production decreasing forage production and the appearance of areas of bare soil. More advanced phases of the degradation process can lead the incidence of invader species and pests in the pastures, which if not correctly managed, may lead situations that are difficult to reverse.

\section{Materials and Methods}

\section{Site description and soil}

The research was carried out in pasture areas in the North of Espírito Santo State, Brazil; between the São Mateus and Itaúnas river basins, including the municipalities of São Mateus, Pinheiros, Boa Esperança, Nova Venécia, Barra de São Francisco, Pedro Canário, Água Doce do Norte and Ecoporanga (Figure 1). The region is in a geomorphic unit defined as Coastal Tablelands (Duarte, 2015) and it is of interest for agricultural use due to its location, topography, extension, climatic conditions and edaphic conditions. The soils of this region are deep and sandy. The soil classes most represented in the Coastal Tablelands are Latosols with 67.5\% and Yellow Acrisols with 25\% (Duarte, 2015). Thus, the study region presented predominantly Latosols and Acrisols.

The climate of the region is considered Tropical (Aw) according to the Köppen classification, with two well-defined seasons: a dry season (winter) with water shortage, from April to September, and a wet season (summer) from October to March. The annual rainfall mean is $1500 \mathrm{~mm}$, the temperature average is between 22 and $27{ }^{\circ} \mathrm{C}$ during the year (Alvares et al., 2013).

\section{Database, sampling protocol and analysis}

Twenty pasture areas with Urochloa brizantha were selected to establish the reference norms. The selection criterion used was the dry matter yield of $U$. brizantha equal to or greater than $15 \mathrm{t} \mathrm{ha}^{-1}$ year ${ }^{-1}$ for forage canopy dry matter. The soil samples were collected on September $9^{\text {th }}$ and October $4^{\text {th }}, 2017$, at a depth of $0-20 \mathrm{~cm}$. Twelve single samples were removed per pasture to compose the compound samples. One hundred and five compound soil samples were collected to measure the soil chemical properties. The chemical analysis was conducted on air dry soil that passed through a 2-mm sieve. After that, the soil fertility factors analyzed were as follows:

organic matter (OM), calcium (Ca), magnesium (Mg), potassium (K), phosphorus $(P)$, sulfur $(S)$, zinc $(Z n)$, boron $(B)$, copper $(\mathrm{Cu})$, iron $(\mathrm{Fe})$, manganese $(\mathrm{Mn})$, base saturation (BS) and cation exchange capacity at $\mathrm{pH} 7$ (CEC), according to the methodology described by Teixeira et al. (2017).

\section{DRIS establishment, sufficiency ranges and diagnostic norms}

A normality test was used to determine the normal distribution for our soil chemical profile data. The data were then used to establish the DRIS soil norms (mean, standard deviation and coefficient of variation) and the sufficiency ranges (mean \pm standard deviation). After this procedure the ratios were calculated, separately, between the data obtained from the soil chemical analysis.

There is little information on DRIS and sufficiency ranges from $U$. brizantha pastures for the region. Thus, it was necessary for comparison to use studies on pastures in the Minas Gerais State and São Paulo (Table 3). Furthermore, these states are close geographically, especially Minas Gerais, the biggest dairy region in Brazil.

In the sequence, the soils of 85 pasture areas were collected randomly at the $0-20 \mathrm{~cm}$ depth to characterize the current nutritional condition of the pastures between São Mateus and Itaúnas river basins. The frequency was analyzed through the occurrence of the characteristics assessed below, within or above of the sufficiency ranges established for the region.

\section{Conclusions}

DRIS norms and sufficiency ranges are established for soils cultivated with $U$. brizantha pastures on Latosols and Acrisols at Coastal Tablelands of Espírito Santo State.

The differences reported between norms of other regions allow confirmation that the norms should be specific for $U$. brizantha pastures and even for soil types.

More than $40 \%$ of the pastures contains $\mathrm{P}, \mathrm{B}, \mathrm{Cu}$, and $\mathrm{Zn}$ concentrations below the indexes recommended and $47 \%$ of the pastures need to apply liming.

\section{Acknowledgments}

The authors thank the Federal University of Espírito Santo (UFES), the Veneza Cooperative, the Espírito Santo Support Foundation for Research, Innovation (FAPES), National Council for Scientific and Technological Development (CNPq) and to the Coordination for the Improvement of Higher Education Personnel (CAPES).

\section{References}

ABIEC - Sumário: Perfil da Agropecuária no Brasil, 2016. Disponível em: http://abiec.com.br/Sumario.aspx. Acesso em: 04 de nov. de 2017.

Alvares CA, Stape IL, Sentelhas PC, Gonçalves JLM, Sparovek G (2013) Köppen's climate classification map for Brazil. Meteorol Z. 22(1): 711-728.

Batista K, Batista FA (2010) Variações nos teores de potássio, cálcio e magnésio em capim-marandu adubado com doses de nitrogênio e de enxofre. Rev Bras Cienc Solo. 34(1): 151-161.

Beaufils ER, Sumner ME (1976) Application of the DRIS approach for calibrating soil, plant yield and plant quality factors on sugarcane. Proceedings of the South Africa Sugar Technology Association. 50(1):118-124. 
Boddey RM, Macedo R, Tarré RM, Ferreira E, Oliveira OC, Rezende CP, Cantarutti RB, Pereira JM, Alves BJR, Urquiaga $S$ (2004) Nitrogen cycling in Brachiaria pastures: the key to understanding the process of pasture decline. Agric Ecosyst Environ. 103(1):389-403.

Camacho MA, Silveira MV, Camargo RA, Natale W (2012) Faixas normais de nutrientes pelos métodos ChM, DRIS e CND e nível crítico pelo método de distribuição normal reduzida para laranjeira-pera. Rev Bras Cienc Solo. 36(1):193-200.

Cavalcanti AC, Oliveira MG, Covre AM, Gontijo I, Braun H, Partelli FL (2017) Primeira aproximação para solo cultivado com Cafeeiro Conilon na região atlântica da Bahia. Coffee Sci. 12(1):316-325.

Costa Junior C, Píccolo M de C, Neto MS, Camargo PB, Cerri CC, Bernoux M (2012) Carbono em agregados do solo sob vegetação nativa, pastagem e sistemas agrícolas no bioma cerrado. Rev Bras Cienc Solo. 36(1):1311-1321.

Deminicies BB, Vieira HD, Silva RF, Abreu JBR, Araújo SAC, Jardim JG (2010) Adubação nitrogenada, potássica e fosfatada na produção e germinação de sementes de capim quicuio-da-amazônia. Rev Bras Sementes. 32(1): 5965.

Dias JRM, Perez DV, Silva LM da, Lemos C de O, Wadt PGS (2010) Normas DRIS para cupuaçuzeiro cultivado em monocultivo e em sistemas agroflorestais. Pesq Agropec Bras. 45(1): 64-71.

Dias JRM, Tucci CAF, Wadt PGF, Silva AM, Santos JZL (2013) Níveis críticos e faixas de suficiência nutricional em laranjeira-pêra na Amazônia Central obtidas pelo método DRIS. Acta Amaz. 43(1): 239-246.

Dow Al, Roberts S (1982) Proposal: critical nutrient ranges for crop diagnosis. Agron J. 74(1): 401-403.

Duarte LRS (2015) Pedôgenese de horizontes com caráter coeso em duas topossequências nos Tabuleiros Costeiros do Ceará. Universidade Federal do Ceará, CE, 54p., 2015.

Embrapa - Empresa Brasileira de Pesquisa Agropecuária. PASTAGEM. Portal Embrapa, 2017. Disponível em: <https://www.embrapa.br/qualidade-da-carne/carnebovina/producao-de-carne-bovina/pastagem $>$. Acesso em: 01 maio 2018.

Garcia RA, Crusciol CAC, Calonego JC, Rosolem CA (2008) Potassium cycling in a corn-brachiaria cropping system. Eur J Agron. 28(1): 579-585.

Guimarães FCN, Serra AP, Marchetti ME, Ensinas SC, Altomar $\mathrm{PH}$, Conrad V do A, Potrich DC, Rosa CBCJ, Martinez MA, Matos FA (2015) Nutrients optimum range (NOR) based on DRIS method to assess the nutritional status of the first ratoon sugarcane. Aust. J. Crop Sci. 9(7):638-645.

Hopkins A, Adamson AH, Bowling PJ (1994) Response of permanent and reseeded grassland to fertilizer nitrogen. 2 . Effects on concentrations of $\mathrm{Ca}, \mathrm{Mg}, \mathrm{K}, \mathrm{Na}, \mathrm{S}, \mathrm{P}, \mathrm{Mn}, \mathrm{Zn}$, $\mathrm{Cu}, \mathrm{Co}$ and $\mathrm{Mo}$ in herbage at a range of sites. Grass Forage Sci. 49(1):9-20.

Kayser M, Isselstein J (2005) Potassium cycling and losses in grassland systems: areview. Grass and Forage Sc. 60,213224.

Marschner H (1995) Mineral Nutrition of Higher Plants. 2ed. San Diego: Academic Press, $889 \mathrm{p}$.

Mesquita MAM, Silveira PM da, Leandro WM, Flores RA, Maranhão DDC (2018) DRIS standards for nutritional evaluation of Phaseolus vulgaris in Cerrado, Goiás state, Brazil. Aust. J. Crop Sci. 12(02):274-280.

Morais NR, Leandro WM, Fernandes EP, Godoy SG (2009) Critérios de interpretação de qualidade do solo para a cultura do algodoeiro produzido do Cerrado Goiano. Biosci J. 25(1): 129-140.

Oladiran O, Olajire F, Abaidoo RC, Ikeorah N (2012) Phosphorus response efficiency in cowpea genotypes. J Agric Sci. 4(1):81-90.

Oliveira IP, Costa KAP, Faquin V, Maciel GA, Neves BP, Machado EL (2009) Efeitos de fontes de cálcio no desenvolvimento de gramíneas solteiras e consorciadas. Ciênc Agrotec. 33(1):592-598.

Oliveira OC, Oliveira IP, Alves BJR, Urquiaga S, Boddey RM (2004) Chemical and biological indicators of decline/ degradation of Brachiaria pastures in the Brazilian Cerrado. Agric Ecosyst Environ. 103(1):289-300.

Partelli FL, Busato JG, Vieira HD, Viana AP, Canellas LP (2009) Qualidade da matéria orgânica e distribuição do fósforo no solo de lavouras orgânicas de café Conilon. Cienc Rural. 39(1): 2065-2072.

Partelli FL, Dias JRM, Vieira HD, Wadt PGS, Paiva Júnior E (2014) Avaliação nutricional de feijoeiro irrigado pelos métodos CND, DRIS e faixas de suficiência. Rev Bras Cienc Solo. 38(1): 858-866.

Partelli FL, Vieira HD, Martins MA (2006) Nutritional diagnosis of the organic Conilon coffee trees (Coffea canephora Pierre ex Froehn): suffiency range approach for leaves and soil. Coffee Sci. 1(1):43-49.

Pimenta LMM, Zonta E, Brasil FC, Anjos LHC, Pereira MG, Stafanato JB (2010) Fertilidade do solo em pastagens cultivadas sob diferentes manejos, no noroeste do Rio de Janeiro. R. Bras. Eng. Agríc. Ambiental. 14(1):1136-1142.

Prezotti LC, Gomes JA, Dadalto GG, Oliveira JÁ (ed) (2007) Manual de Recomendação de Calagem e Adubação para o Estado do Espírito Santo - 5a aproximação. Vitória, Espírito Santo State. 305.

Raij B Van, Cantarella H, Quaggio JA, Furlani AMC (ed) (1997) Recomendações de adubação e calagem para o Estado de São Paulo. Campinas-Instituto Agronômico de Campinas. 285.

Ribeiro AC, Guimarães PTG, Alvarez VVH (ed) (1999) Recomendações para uso de corretivos e fertilizantes em Minas Gerais. 5a Aproximação. Viçosa, Minas Geais State. Comissão de Fertilidade do Solo do Estado de Minas Gerais. 359.

Rocha AC, Leandro WM, Rocha AO, Santana JG, Andrade JWS (2007) Normas DRIS para cultura do milho semeado em espaçamento reduzido na região de Hidrolândia, GO, Brasil. J Biosci. 23(1): 50-60.

Santana JG, Leandro WM, Naves RV, Cunha PP da (2008) Normas DRIS para interpretação de análises de folha e solo, em laranjeira pêra, na região central de Goiás. Pesq Agropec Trop. 38(1): 109-117.

Santos CCK, Bini D, Goulart Filho T, Knob A, Medina CC, Andrade Filho G, Nogueira, MA (2015) Reclamation status of a degraded pasture based on soil health indicators. Scientia Agricola. 72(1):195-202.

Silva JTA, Silva IP, Rodas CL, Carvalho JG (2013) Critical levels for Brachiaria brizantha and Panicum maximum using different sources of phosphorus. Idesia. 31(1):59-65.

Sofi JA, Rattan RK, Kirmani NA, Chesti MH, Bisati I (2017) Diagnosis and recommendation integrated system approach for major and micronutrient diagnostic norms for apple (Malus Domestica Borkh) under varying ages and management practices of apple orchards. J Plant Nut, 40(12):1784-1796.

Sousa LF, Maurício RM, Moreira GR, Gonçalves LC, Borges I, Pereira LGR (2010) Nutritional evaluation of "Braquiarão" 
grass in association with "Aroeira" trees in a silvopastoral system. Agroforest Syst. 79(1):189-199.

Steiner F, Lana MC, Zoz T, Frandoloso JF, Fey R (2012) Extraction methods and availability of phosphorus for soybean in soils from Paraná State, Brazil. Semina: Ciências Agrárias. 33(1):1005-1014.

Teixeira PC, Donagemma GK, Fontana A, Teixeira WG (2017). Manual de métodos de análise de solo. 3rd edn. Brasília-DF: Embrapa, 573b.
Tiwari RJ (2006) Response of sugarcane (Saccharum officinarum) to direct and residual effect of sulphur. Indian J Agric Sci, 76(1): 117-119.

Veneklaas EJ, Lambers H, Bragg J, Finnegan PM, Lovelock CE, Plaxton WC, Price CA, Scheible WR, Shane MW, White PJ, Raven JA (2012) Opportunities for improving phosphorususe efficiency in crop plants. New Phytol. 195(2): 306-320.

Whitehead DC (2000). Nutrient elements in grassland: Soilplant-animal relationships. Wallingford: CAB International, 369. 